\title{
NMDA Receptor Plasticity in the Perirhinal and Prefrontal Cortices Is Crucial for the Acquisition of Long-Term Object-in-Place Associative Memory
}

\author{
Gareth R. I. Barker and E. Clea Warburton \\ Medical Research Council Centre for Synaptic Plasticity, Department of Anatomy, University of Bristol, Bristol BS8 1 TD, United Kingdom
}

\begin{abstract}
A key process for recognition memory is the formation of associations between an object and the place in which it was encountered, a process that has been shown to require the perirhinal (PRH) and medial prefrontal (mPFC) cortices. Here we demonstrate, for the first time, the importance of glutamatergic neurotransmission, within the PRH and $\mathrm{mPFC}$, for object-in-place associative recognition memory. Unilateral blockade of AMPA receptors (by CNQX) in the PRH and MPFC in opposite hemispheres impaired an object-in-place task in rats, confirming that these cortical regions operate within a neural network for object-in-place recognition memory. Intra-mPFC infusions of AP5 (NMDA receptor antagonist) impaired short-term memory and the acquisition of long-term memory, but had no effect on retrieval. AP5 infusions into the PRH disrupted acquisition of long-term memory, but not short-term memory or retrieval. Significantly, crossed AP5 infusions into both the PRH and mPFC disrupted acquisition of long-term memory but were without effect on short-term memory. Finally a unilateral infusion of the selective kainate $\left(\mathrm{GLU}_{\mathrm{K} 5}\right)$ receptor antagonist UBP302 [(S)-1-(2-amino-2carboxyethyl)-3-(2-carboxybenzyl)pyrimidine-2,4-dione] into the PRH combined with a unilateral infusion of AP5 into the contralateral mPFC significantly impaired short-term object-in-place associative memory. These data show that the PRH and mPFC make distinct contributions to object-in-place associative memory and that the encoding of long-term but not short-term memory requires concurrent NMDA receptor activation in both cortical regions. In contrast, short-term object-in-place memory appears to be dependent on kainate receptor activation in the PRH and NMDA receptor activation in the $\mathrm{MPFC}$.
\end{abstract}

Key words: recognition memory; perirhinal cortex; prefrontal cortex; object-in-place; glutamate receptors; rat

\section{Introduction}

Recognition memory is a fundamental explicit memory process involving judgments of the prior occurrence of stimuli. Such judgments may be made by either using the relative familiarity of individual objects (familiarity discrimination) and/or by using associations between objects and the place in which they occur (object-in-place associative memory) (Gaffan and Parker, 1996; Dix and Aggleton, 1999), as well as by using associations between objects and context (Dix and Aggleton, 1999) or recency information (Mitchell and Laiacona, 1998; Barker et al., 2007).

Recently we have shown that that familiarity discrimination and object-in-place associative memory may be differentially affected by damage to the medial prefrontal (mPFC) or perirhinal (PRH) cortices (Barker et al., 2007). Thus, damage to the mPFC impairs object-in-place memory, but not familiarity discrimination, whereas damage to the PRH impairs both these recognition memory processes, a finding supported by other reports in the

Received Sept. 28, 2007; revised Jan. 25, 2008; accepted Jan. 25, 2008.

This work was supported by a project grant from the Biotechnology and Biological Sciences Research Council. We thank Malcolm Brown for his help and comments on this manuscript, Jane Robbins and Katherine Narduzzo for assistance with histology, and Dr. Andy Doherty for help with the figures.

Correspondence should be addressed to Dr. E. Clea Warburton, Medical Research Council Centre for Synaptic Plasticity, Department of Anatomy, University of Bristol, Bristol BS8 1 TD, UK. E-mail: e.c.warburton@bristol.ac.uk. DOI:10.1523/JNEUROSCI.4447-07.2008

Copyright $\odot 2008$ Society for Neuroscience $\quad$ 0270-6474/08/282837-08\$15.00/0 literature (Gaffan and Murray, 1992; Ennaceur et al., 1996; Murray and Bussey, 1999; Bussey et al., 2000). Importantly it has also been shown that these cortical regions must operate within an integrated neural network to make successful discriminations using object-in-place association information (Browning et al., 2005; Barker et al., 2007).

Although we have identified the critical neural circuitry for object-in-place recognition memory, the neurochemical bases for the acquisition of this memory process have not been defined. We and others have previously shown that blockade of NMDA receptor (NMDAR) neurotransmission using the competitive antagonist AP5 (Watkins et al., 1990) in the PRH significantly impairs familiarity discrimination, after long $(24 \mathrm{~h})$ but not short (20 min) retention delays (Winters and Bussey, 2005; Barker et al., 2006), whereas short-term familiarity discrimination is dependent on kainate receptor (KAR) activation (Barker et al., 2006). Here we examine whether acquisition or retrieval of object-in-place associative memory requires glutamate neurotransmission in the PRH, mPFC, or the PRH-mPFC circuit. First, we examined the contribution of AMPAR neurotransmission within the PRH, mPFC, or both regions for object-in-place associative memory using CNQX, an antagonist of AMPAR. Second, we examined whether acquisition or retrieval of object-inplace memory requires NMDARs in the PRH or mPFC by testing object-in-place memory after both short $(5 \mathrm{~min})$ and long $(1 \mathrm{~h})$ 
retention delays. We then examined whether acquisition of this memory process requires concurrent NMDAR activation in both brain regions and demonstrated that such activation was only necessary for long-term memory. In light of this finding, we examined whether short-term object-in-place memory is dependent on KAR activation in the PRH. Finally, we conducted a novel object preference test to confirm that NMDAR blockade in the PRH impairs acquisition but not retrieval of familiarity discrimination after a $1 \mathrm{~h}$ delay, because our previous studies have only used delays of $20 \mathrm{~min}$ or $24 \mathrm{~h}$ (Barker et al., 2006).

\section{Materials and Methods}

All experiments were conducted in male pigmented rats (DA strain; weighing 200-250 g at the start of the experiments; Bantin and Kingman, Hull, UK). The animals were housed, in pairs, under a $12 \mathrm{~h} \mathrm{light/dark}$ cycle (light phase, 6:00 P.M. to 06:00 A.M.). Behavioral training and testing were conducted during the dark phase of the cycle. Food and water were available ad libitum throughout the experiment. All animal procedures were performed in accordance with United Kingdom Animals Scientific Procedures Act (1986) and associated guidelines. All efforts were made to minimize any suffering and the number of animals used. All statistical analyses used a significance level of 0.05 .

\section{Surgery}

Each rat was anesthetized with isoflurane (induction 4\%, maintenance $2-3 \%)$. The rat was secured in a stereotaxic frame with the incisor bar set at $3.3 \mathrm{~mm}$ below the interaural line to achieve a flat skull. Rats were implanted with bilateral cannulas aimed at either the PRH or mPFC or into both regions. Two stainless-steel guide cannulas (26 gauge; Plastics One, Semat, UK) were implanted bilaterally through burr holes in the skull at the following coordinates relative to skull at bregma: $\mathrm{PRH}$ : anterior-posterior $(\mathrm{AP}),-5.6 \mathrm{~mm}$; mediolateral $(\mathrm{ML}), \pm 4.47 \mathrm{~mm}$; dorsoventral (DV), $-6.7 \mathrm{~mm}$ (relative to surface of the skull) with the manipulator arm at an angle of $20^{\circ}$ to the vertical; mPFC: AP, $+3.20 \mathrm{~mm}$; ML, $\pm 0.75 \mathrm{~mm}$; DV,$-3.5 \mathrm{~mm}$. The cannulas were anchored to the skull by stainless-steel skull screws (Plastics One) and dental acrylic. After surgery, each animal was given fluid replacement therapy ( $5 \mathrm{ml}$ of saline, s.c.) and analgesia $(0.05 \mathrm{ml}$ of Temgesic, i.m. $)$ and was housed individually. The animals were allowed to recover for at least $10 \mathrm{~d}$ before habituation to the testing arena began. Between infusions, 33 gauge obdurators (Plastics One) were used to keep the cannulas patent.

\section{Infusions}

General procedures followed Barker et al. (2006). The drugs used were as follows: 6-cyano-7-nitroquinoxaline (CNQX disodium salt; Tocris Bioscience, Bristol, UK), 2-amino-5-phosphonopentanoic acid (AP5; Tocris Bioscience), and (S)-1-(2-amino-2-carboxyethyl)-3-(2-carboxybenzyl)pyrimidine-2,4-dione (UBP302; Tocris Bioscience). CNQX and AP5 were dissolved in sterile $0.9 \%$ saline solution. Vehicle infusions consisted of saline. AP5 was infused at a concentration of $5 \mu \mathrm{g} / \mathrm{side}$, CNQX at 2.5 $\mu \mathrm{g} / \mathrm{side}$, and UBP302 at $0.5 \mu \mathrm{g} / \mathrm{side}$. Infusions were made into the cortex through a 33 gauge cannula (Plastics One) inserted into the implanted guide cannulas and attached to a $5 \mu \mathrm{l}$ Hamilton syringe via polyethylene tubing. A volume of $1.0 \mu \mathrm{l}$ was injected into each hemisphere over a 2 min period by infusion pump (Harvard Bioscience, Holliston, MA). The infusion cannulas remained in place for an additional $5 \mathrm{~min}$.

\section{Histology}

At the end of the experiment, each rat was anesthetized with Euthetal (Rhône Mérieux, Toulouse, France) and perfused transcardially with PBS followed by $4 \%$ paraformaldehyde. The brain was postfixed in paraformaldehyde for a minimum of $2 \mathrm{~h}$ before being transferred to $30 \%$ sucrose in $0.2 \mathrm{M}$ phosphate buffer and left for $48 \mathrm{~h}$. Coronal sections were cut at $40 \mu \mathrm{m}$ on a cryostat and stained with cresyl violet. Cannula locations were checked against standardized sections of the rat brain. Histological examination confirmed that all rats in the PRH group had the tip of the bilateral cannulas in the PRH (Shi and Cassell, 1999), and all rats in the $\mathrm{mPFC}$ group had needle tips located in the ventral portion of prelimbic or dorsal portion of the infralimbic region of the prefrontal cortex
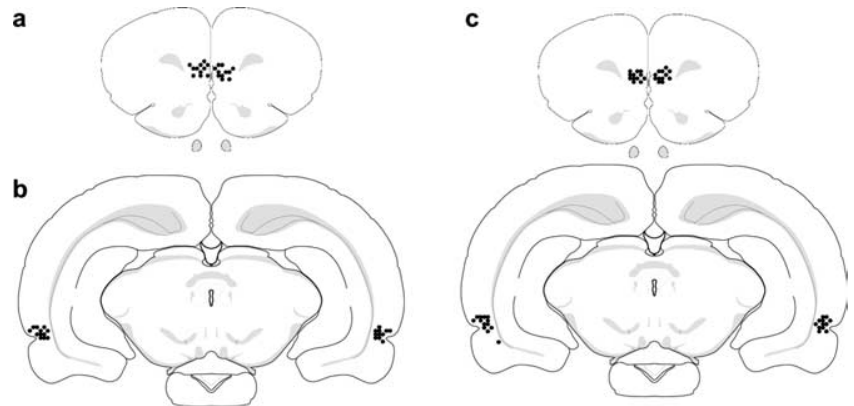

Figure 1. Diagrammatic representations of the individual infusion sites in each animal. $\boldsymbol{a}$, Bilateral mPFC group. $\boldsymbol{b}$, Bilateral PRH group. $\boldsymbol{c}, \mathrm{PRH}+\mathrm{mPFC}$ group. All of the infusion sites were within the PRH or mPFC.

(Fig. 1). From unpublished observations in the laboratory, using Indian ink and biotinylated oligonucleotides for visualization, the region infused is estimated to be $1-1.5 \mathrm{~mm}^{3}$, with the region being largely confined to perirhinal cortex or the prelimbic/infralimbic regions of the prefrontal cortex. This spread is consistent with previously quoted results in other brain regions (Martin, 1991; Izquierdo et al., 2000; Attwell et al., 2001).

\section{Apparatus}

Exploration occurred in an open-topped arena $(50 \times 90 \times 100 \mathrm{~cm})$ made of wood. The walls inside the arena (painted black) were surrounded with a black cloth to a height of $1.5 \mathrm{~m}$ on one side to conceal the experimenter, and the floor of the arena was covered with sawdust. An overhead camera and a video recorder were used to monitor and record the animal's behavior for subsequent analysis. The stimuli presented were copies of objects composed of "Duplo" (Lego UK, Slough, UK) that varied in shape, color, and size $(9 \times 8 \times 7 \mathrm{~cm}$ to $25 \times 15 \times 10 \mathrm{~cm})$ and were too heavy for the animal to displace.

\section{Behavioral testing}

Pretraining. After being handled for a week, the animals were habituated to the arena without stimuli for $10-15$ min daily for $4 \mathrm{~d}$ before the commencement of the behavioral testing; the animals were also habituated to the infusion procedure.

Object-in-place task (experiments 1-4). This task comprised a sample phase and a test phase separated by either a 5 min or a $1 \mathrm{~h}$ delay. In the sample phase, the subjects were presented with four different objects (A, $\mathrm{B}, \mathrm{C}$, and D). These objects were placed in the corners of the arena $15 \mathrm{~cm}$ from the walls (Fig. 2a). In this experiment, one of the walls of the arena was gray in color, whereas the other three remained black, and the curtains were removed from around the arena to provide additional extramaze cues. Each subject was placed in the center of the arena and allowed to explore the objects for $5 \mathrm{~min}$. During the delay period, all the objects were cleaned with alcohol to remove olfactory cues and any sawdust that had stuck to the object. In the test phase, two of the objects, e.g., $\mathrm{B}$ and $\mathrm{D}$ (which were both on the left or right of the arena), exchanged positions, and the subject were allowed to explore the objects for $3 \mathrm{~min}$. The time spent exploring the two objects that had changed position was compared with the time spent exploring the two objects that had remained in the same position. The objects moved (i.e., those on the left or right) and the position of the objects in the sample phase were counterbalanced between rats. If object-in-place memory is intact, the subject will spend more time exploring the two objects that are in different locations, compared with the two objects that are in the same locations.

Novel object preference task (experiment 5). This procedure comprised an acquisition phase separated by a delay from a recognition test. In the acquisition phase, duplicate copies of an object (e.g., A1 and A2) were placed near the two corners at either end of one side of the arena $(10 \mathrm{~cm}$ from each adjacent wall) (Fig. $2 b$ ). The animal was placed into the arena facing the center of the opposite wall and allowed a total of either $40 \mathrm{~s}$ of exploration of A1 and A2, or 4 min in the arena. Exploratory behavior was defined as the animal directing its nose toward the object at a distance 
a. Object-in-place

Sample

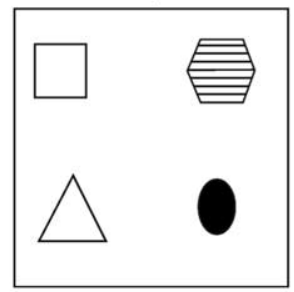

b. Novel object preference

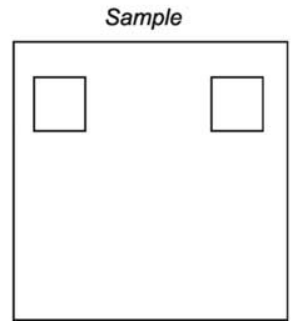

Figure 2. Diagram of the two object-recognition memory tasks. $\boldsymbol{a}$, 0bject-in-place task. $\boldsymbol{b}$ Novel object preference task.

of $<2 \mathrm{~cm}$. Other behaviors, such as looking around while sitting on or resting against the object, were not considered as exploration. The delay between the phases was $5 \mathrm{~min}$ or $1 \mathrm{~h}$ depending on the experiment. At test ( 3 min duration), the animal was replaced in the arena, presented with two objects using the same positions as at acquisition: one object (A3) was the third copy of the object used in the sample phase, and the other was a novel object (B3). The positions of the objects in the test and the objects used as novel or familiar were counterbalanced between the animals in a group and between the control and drug-treated groups.

Design

A different group of rats was used to investigate the effects of glutamate receptor antagonists in the $\mathrm{PRH}$ or $\mathrm{mPFC}$. A third group of animals had bilateral cannulas implanted into both the $\mathrm{PRH}$ and $\mathrm{mPFC}$ $(\mathrm{PRH}+\mathrm{mPFC})$. This group received a combined unilateral infusion into the PRH and mPFC in either the same (ipsi) or opposite (contra) hemisphere. CNQX, UBP302, or vehicle was infused starting $15 \mathrm{~min}$ before the commencement of the acquisition phase, and after a minimum of $48 \mathrm{~h}$, drug or vehicle was infused in a cross-over design and the animal was tested again. AP5 or vehicle was infused either $15 \mathrm{~min}$ before the acquisition phase (as for CNQX) or 15 min before the test phase.

When testing the effects of dual infusions in the PRH+mPFC, the animals that received drug infusions into the same hemisphere (ipsi) on day 1 received infusions into opposite hemispheres (contra) on day 2. Likewise, the animals in the contra group on day 1 received ipsilateral infusions on day 2. All rats in the $\mathrm{PRH}+\mathrm{mPFC}$ group were run in the experiments in the same order.

The initial group sizes $(n)$ were as follows: $\mathrm{PRH}, n=12$; $\mathrm{mPFC}, n=11$; $\mathrm{PRH}+\mathrm{mPFC}, n=12$. Cannula blockage resulted in the occasional loss of an animal (as indicated by reduced degrees of freedom in the quoted statistical tests).

Data analysis

All measures of exploration were made with the experimenter blind to the drug status of each animal. Exploratory behavior was defined as the animal directing its nose toward the object at a distance of $<2 \mathrm{~cm}$. Any other behavior, such as looking around while sitting on or resting against the object, was not considered as exploration. Any subjects that failed to complete a minimum of $15 \mathrm{~s}$ exploration in the sample phase or 10 seconds of exploration in the test phase were excluded from the analysis. Discrimination between the objects was determined using a discrimination ratio, calculated as the difference in time spent by each animal ex-

\section{Object-in-place}

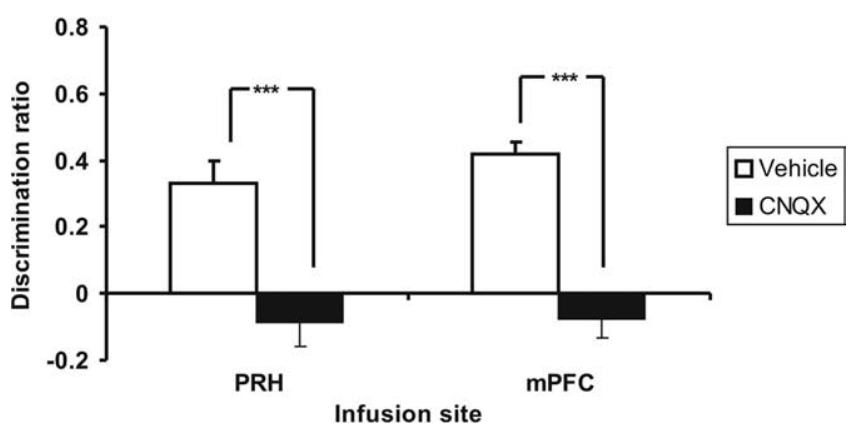

Figure 3. Object-in-place task. Infusion of CNQX into the PRH or mPFC significantly impaired discrimination between the objects that had exchanged location at test and those that remained in the same location. Illustrated for each group is the mean + SEM discrimination ratio. ${ }^{* * *} p<0.001$, difference between groups.

ploring the novel compared with the familiar object divided by the total time spent exploring both objects. This measure therefore takes into account individual differences in the total amount of exploration between rats (Ennaceur and Delacour, 1988; Dix and Aggleton, 1999). Comparisons were made using a multifactor ANOVA followed by post hoc pairwise comparisons, with a Bonferroni correction for multiple comparisons. Additional analyses in both experiments examined whether individual groups had discriminated between the objects, using a within-subjects $t$ test (two-tailed) comparing the discrimination ratio against chance performance.

\section{Results}

\section{Experiment 1}

In this experiment, we investigated the relative importance of AMPAR-mediated neurotransmission in the PRH and mPFC in object-in-place associative memory. The antagonist of AMPARs CNQX was infused into either the PRH or the mPFC via chronically indwelling cannulas before the sample phase of an objectin-place task, and the animals were tested after a 5 min delay. Next, to examine the importance of intrahemispheric interactions between the PRH and MPFC in object-in-place performance, the effects of unilateral CNQX infusions into the PRH and $\mathrm{mPFC}$ in the same hemisphere (ipsi group) were compared with the effects of infusions into the PRH in one hemisphere and the $\mathrm{mPFC}$ in the other hemisphere (contra group).

\section{Recognition during the test phase}

Animals receiving bilateral vehicle infusions into either the $\mathrm{PRH}$ or the $\mathrm{mPFC}$ spent longer exploring the objects that were in a different location compared with the objects that were in the same location. In contrast, bilateral infusions of CNQX into the $\mathrm{PRH}$ or mPFC significantly impaired performance. Two-way ANOVA [within-subject factor, drug (vehicle vs CNQX); between-subjects factor, region (PRH vs $\mathrm{mPFC}$ )] confirmed that infusions of CNQX significantly disrupted object-in-place performance (drug, $F_{(1,19)}=78.71, p<0.001$ ) independent of the region into which it was infused (region, $F_{(1,19)}=0.246, p<0.05$; interaction, $\left.F_{(1,19)}=0.530, p>0.05\right)$. Thus, CNQX infused into either the PRH $\left(F_{(1,10)}=25.55, p<0.001\right)$ or $\operatorname{mPFC}\left(F_{(1,9)}=\right.$ 52.53, $p<0.001)$ significantly impaired performance compared with infusions of vehicle (Fig. 3). Further analyses confirmed that rats receiving a vehicle infusion into the $\mathrm{PRH}$ or $\mathrm{mPFC}$ showed significant discrimination $\left(t_{(10)}=4.66, p=0.01\right.$ and $t_{(9)}=10.58$, $p<0.01$, respectively). However infusions of CNQX into the $\mathrm{PRH}$ or mPFC markedly impaired discrimination $\left(t_{(10)}=1.10\right.$, $p>0.05$ and $t_{(9)}=1.26, p>0.05$, respectively). 
Table 1. Experiment 1: mean exploration times \pm SEM during the sample or test phase after infusions of vehicle or CNQX into the mPFC or PRH in the object-in-place task (5 min delay)

\begin{tabular}{|c|c|c|c|c|c|c|}
\hline \multirow[b]{2}{*}{ Drug/side } & \multicolumn{2}{|l|}{$\mathrm{mPFC}$} & \multicolumn{2}{|l|}{ PRH } & \multicolumn{2}{|l|}{ Unilateral mPFC and PRH } \\
\hline & Expl. in sample phase (s) & Expl. in test phase (s) & Expl. in sample phase (s) & Expl. in test phase (s) & Expl. in sample phase (s) & Expl. in test phase (s) \\
\hline CNQX & $62.5 \pm 2.1$ & $23.5 \pm 2.1$ & $57.9 \pm 3.9$ & $18.7 \pm 2.1$ & & \\
\hline Veh & $57.3 \pm 3.0$ & $14.2 \pm 1.5$ & $60.7 \pm 3.6$ & $18.2 \pm 1.5$ & & \\
\hline Ipsi & & & & & $50.7 \pm 4.3$ & $18.8 \pm 3.05$ \\
\hline Contra & & & & & $48.8 \pm 5.3$ & $17.9 \pm 1.60$ \\
\hline
\end{tabular}

Expl., Exploration; Veh, vehicle.

Unilateral infusions of CNQX into the PRH and $\mathrm{mPFC}$ in the same hemisphere (CNQX ipsi), regardless of whether it was the left or right hemisphere, had no detrimental effect on the animal's ability to discriminate between objects in a new location compared with objects in the old location (mean discrimination ratio $\pm \mathrm{SEM}, 0.31 \pm 0.064)$. In contrast, infusions into the $\mathrm{PRH}$ and $\mathrm{mPFC}$ in opposite hemispheres (CNQX contra) produced a significant impairment in performance (mean discrimination ratio, $-0.035 \pm 0.05)$. Within-subjects ANOVA revealed a significant difference between the groups $\left(F_{(1,9)}=16.77, p<0.01\right)$. Further analyses confirmed that the CNQX ipsi group showed significant discrimination between the objects that had exchanged location at test compared with those objects that remained in the same location, whereas the CNQX contra group did not $\left(t_{(9)}=4.62, p=0.01\right.$ and $t_{(9)}=0.65, p<0.05$, respectively). Thus concomitant glutamatergic neurotransmission via AMPAR in the PRH and mPFC is necessary for the performance of an object-in-place task.

\section{Exploration in sample and test phases}

Analysis of the total amount of exploration completed in the sample phase after CNQX or vehicle infusions into the PRH or mPFC revealed no significant differences (sample phase: main effect of drug, $F_{(1,19)}=0.235, p>0.05$; main effect of region, $F_{(1,19)}=0.19, p>0.05$; drug $\times$ region interaction, $F_{(1,19)}=2.66$, $p>0.05)$. Analysis of the total amount of exploration in the test phase after drug or vehicle infusions into the $\mathrm{PRH}$ or $\mathrm{mPFC}$ revealed a significant drug-by-region interaction $\left(F_{(1,19)}=6.70\right.$, $p<0.05)$ and a significant main effect of drug $\left(F_{(1,19)}=8.26\right.$, $p=0.01)$; the main effect of region was not significant $\left(F_{(1,19)}\right.$ $=0.026, p>0.05)$. Further analysis of the total exploration in test after infusion into the $\mathrm{MPFC}$ revealed a significant difference between CNQX and vehicle $\left(F_{(1,9)}=12.19, p<0.01\right)$. Inspection of the mean exploration data (Table 1 ) showed that infusions of CNQX into the mPFC resulted in a greater amount of object exploration in the test phase compared with infusions of vehicle.

Analysis of the amount of exploration completed after infusions into the same hemisphere compared with infusions in opposite hemispheres in the sample and test phases revealed no significant differences (sample phase: $F_{(1,9)}=0.08, p>0.05$; test phase $\left.F_{(1,9)}=0.189, p>0.05\right)$. The mean exploration data are presented in Table 1.

\section{Experiment 2}

Experiment 2 investigated whether NMDAR-mediated neurotransmission in the PRH or $\mathrm{mPFC}$ was required for (1) shortterm $(5 \mathrm{~min})$ or long-term $(1 \mathrm{~h})$ object-in-place associative memory; (2) retrieval of long-term object-in-place associative memory; and (3) whether concurrent NMDAR neurotransmission in the PRH and $\mathrm{mPFC}$ is necessary for short- or long-term object-in-place associative memory. First, the functional effects of NMDAR blockade were tested by infusing the NMDA antag- onist AP5 bilaterally into either the PRH or mPFC, either before the sample phase or before the test phase. To assess whether concurrent NMDAR neurotransmission in the PRH and $\mathrm{mPFC}$ is required for object-in-place memory, AP5 was infused unilaterally into both the PRH and $\mathrm{mPFC}$ in either the same hemisphere (ipsi) or in opposite hemispheres (contra). Hence, in this experiment, there was no vehicle condition, because the ipsi group served as the control condition.

\section{Importance of NMDAR-mediated neurotransmission in short-} term memory or encoding of long-term memory

Blockade of NMDAR neurotransmission in the MPFC before the sample phase impaired object-in-place associative memory independent of the retention delay (Fig. 4ai), whereas blockade of NMDAR neurotransmission in the PRH impaired object-inplace performance after a long but not a short delay (Fig. 4aii). This pattern of results was confirmed statistically, by a significant drug $\times$ region interaction $\left(F_{(1,33)}=6.75, p<0.05\right)$ and a significant region $\times$ delay interaction $\left[F_{(1,33)}=5.22, p<0.05\right.$; the drug $X$ region $\times$ delay interaction failed to reach significance $\left.\left(F_{(1,33)}=2.12, p>0.05\right)\right]$. Further analyses of the effects of AP5 into each region independently showed that infusions into the mPFC significantly impaired discrimination at both retention delays (ANOVA, drug, $F_{(1,13)}=27.65, p<0.001$; delay, $F_{(1,13)}=$ $0.00, p>0.05$; interaction, $\left.F_{(1,13)}=0.062, p>0.05\right)$, whereas infusions into the $\mathrm{PRH}$ only impaired discrimination depending on the delay (ANOVA, drug, $F_{(1,20)}=5.67, p<0.05$; delay, $F_{(1,20)}$ $=14.55, p<0.001$; interaction, $\left.F_{(1,20)}=6.90, p<0.05\right)$. Analysis of the simple main effects confirmed that the detrimental effect of AP5 in the PRH occurred specifically after the long retention delay $\left(1 \mathrm{~h}\right.$ delay, $F_{(1,10)}=11.64, p<0.01 ; 5$ min delay, $F_{(1,10)}=$ $0.13, p>0.05)$.

Further analyses showed that at both a 5 min and $1 \mathrm{~h}$ delay, intra-mPFC vehicle infusions produced significant discrimination in the object-in-place task $t_{(8)}=4.93, p=0.01$ and $t_{(5)}=$ $4.29, p<0.01$, respectively). In contrast, rats infused with AP5 into the $\mathrm{mPFC}$ did not show significant discrimination at either delay ( $\left.5 \mathrm{~min}, t_{(8)}=0.108, p>0.05 ; 1 \mathrm{~h}, t_{(5)}=0.31, p>0.05\right)$.

At both a $5 \mathrm{~min}$ and $1 \mathrm{~h}$ delay, intra-PRH infusions of vehicle produced significant object-in-place discrimination $\left(t_{(10)}=5.65\right.$, $p<0.01$ and $t_{(10)}=4.90, p=0.01$, respectively). In contrast, rats infused with AP5 into the PRH showed significant discrimination after the 5 min delay, but not after the $1 \mathrm{~h}$ delay $\left(t_{(10)}=5.70, p<\right.$ 0.01 and $t_{(10)}=0.016, p>0.05$, respectively).

Importance of NMDAR-mediated neurotransmission in retrieval Blockade of NMDAR neurotransmission either in the MPFC or $\mathrm{PRH}$ before retrieval after a $1 \mathrm{~h}$ delay produced no significant impairments in object-in-place performance (Fig. $4 b$ ), confirmed by a nonsignificant main effect of drug $\left(F_{(1,14)}=0.154\right.$, $p=0.70)$ and nonsignificant drug $\times$ area interaction $\left(F_{(1,14)}=\right.$ $0.123, p=0.73)$. Further analyses showed that rats infused with vehicle or AP5 into the PRH or mPFC showed significant dis- 


\section{Object-in place task - encoding}

ai

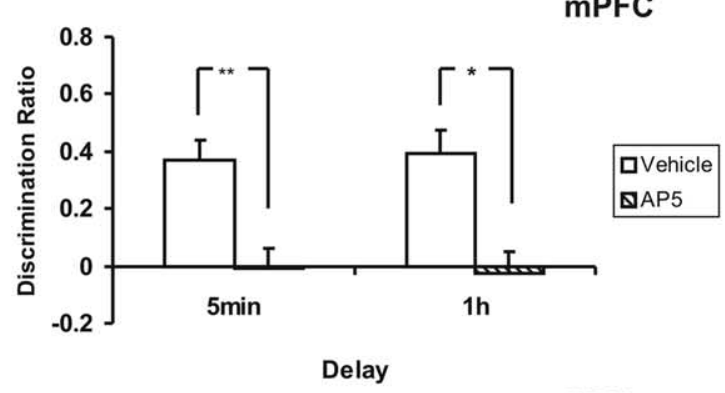

aii

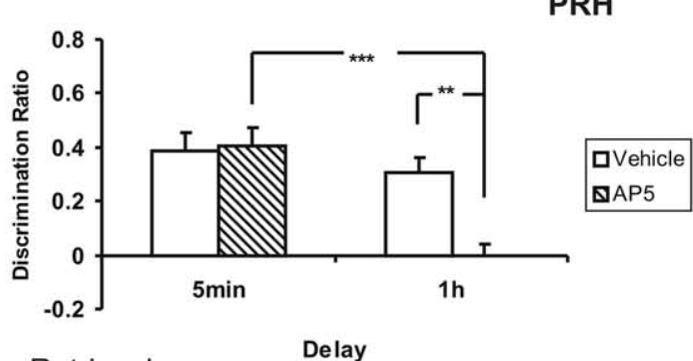

b

$$
\text { Retrieval }
$$

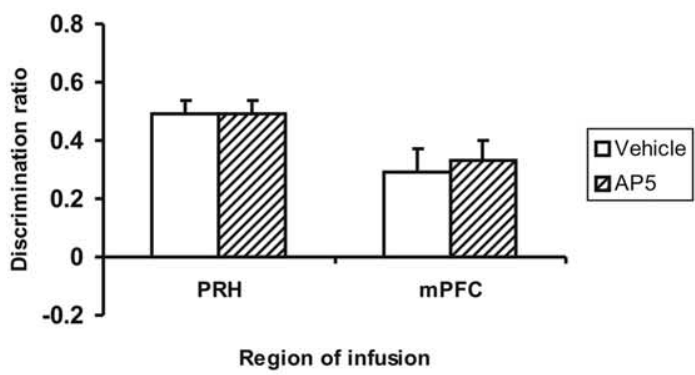

C

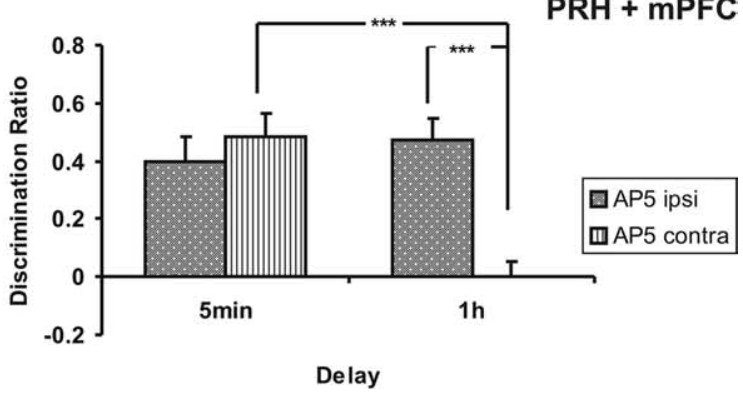

Figure 4. Object-in-place task. ai, AP5 infusions into the $\mathrm{mPFC}$ before the sample phase significantly impaired object-in-place performance after a 5 min or 1 h delay. ${ }^{*} p<0.05,{ }^{* *} p<$ 0.01, difference between groups. aii, AP5 infusions into the PRH before the sample phase impaired object-in-place performance after a $1 \mathrm{~h}$ delay, but not a 5 min delay. ${ }^{* *} p<0.01$, ${ }^{* * *} p<0.001$, difference between groups. $\boldsymbol{b}$, AP5 infusions into the PRH or mPFC before the test phase had no effect on object-in-place performance after a $1 \mathrm{~h}$ delay. c, Unilateral AP5 infusions into the PRH and mPFC in opposite hemispheres (AP5 contra) impaired object-inplace performance after a $1 \mathrm{~h}$ delay, but not a 5 min delay. AP5 infusion into the PRH and $\mathrm{mPFC}$ in the same hemisphere (AP5 ipsi) has no effect on performance. ${ }^{* * *} p<0.001$, difference between groups.

crimination between the objects that had moved location compared with objects in the same location ( $\mathrm{PRH}$ saline, $t_{(7)}=8.71$, $p<0.0001 ;$ PRH AP5, $t_{(7)}=8.83, p<0.0001 ;$ mPFC saline, $t_{(7)}=$ 3.27, $\left.p=0.01 ; \mathrm{mPFC} \mathrm{AP5}, t_{(7)}=4.15, p<0.01\right)$.

Importance of concurrent NMDAR-mediated neurotransmission in the PRH and $m P F C$

Unilateral AP5 infusions into the $\mathrm{PRH}$ and $\mathrm{mPFC}$ produced a significant effect on performance, as a function of group (ipsi vs contra) and the retention delay used (ANOVA, group $\times$ delay interaction, $F_{(1,18)}=11.46, p<0.01$; group, $F_{(1,18)}=5.27, p<$ 0.05 ; delay, $\left.F_{(1,18)}=8.05, p<0.05\right)$ Further analysis of the simple main effects showed that the contra group were impaired after the long retention delay $\left(F_{(1,9)}=20.50, p<0.001\right)$, but not after the short retention delay $\left(F_{(1,9)}=0.49, p>0.05\right)$. The ipsi group were not impaired at any delay (Fig. $4 c$ ).

Further analyses revealed that after a 5 min delay, both the AP5 ipsi and AP5 contra groups showed significant discrimination $\left(t_{(9)}=4.15, p<0.01\right.$ and $t_{(9)}=5.98, p<0.01$, respectively). In contrast, after the $1 \mathrm{~h}$ delay, animals in the AP 5 contra group did not show significant discrimination $\left(t_{(9)}=0.022, p>0.05\right)$, whereas the AP5 ipsi group $\operatorname{did}\left(t_{(9)}=6.23, p<0.01\right)$.

Thus, NMDAR neurotransmission in the PRH-mPFC circuit is selectively required for long-term but not short term object-inplace recognition memory.

Exploration in sample and test phases

Analysis of the amount of exploration completed in the sample phases after presample infusion of AP5 into either the PRH or $\mathrm{mPFC}$ prior revealed no significant differences whether a $5 \mathrm{~min}$ or 1 h delay was used $\left(\right.$ drug $\times$ region $\times$ delay, $F_{(1,33)}=0.60, p>$ 0.05). Similarly, no significant differences were found in the amount of total exploration completed in the test phase (drug $X$ region $\times$ delay, $\left.F_{(1,33)}=0.013, p>0.05\right)$.

Analysis of the amount of exploration completed after ipsi AP5 infusions compared with contra AP5 infusions in opposite hemispheres in the sample and test phases revealed no significant differences whether a 5 min or $1 \mathrm{~h}$ delay was used (sample phase, group $\times$ delay, $F_{(1,18)}=0.044, p>0.05$; test phase, $F_{(1,18)}=$ $0.045, p>0.05)$. The mean exploration data are presented in Table 2 .

\section{Experiment 3}

Experiment 2 showed that long-term but not short-term objectin-place associative memory requires concurrent NMDA receptor activation in both the PRH and the $\mathrm{MPFC}$, a pattern of results that suggests that a non-NMDA receptor-dependent process in the PRH may be sufficient to establish the necessary memory, even in the absence of the contralateral mPFC. To test this hypothesis CNQX was infused unilaterally into the $\mathrm{PRH}$, and at the same time AP5 was infused into the MPFC in either the same (CNQX+AP5 ipsi group) or the opposite (CNQX+AP5 contra group) hemisphere. All infusions were given before the sample phase, and object-in-place memory was tested after a 5 min retention delay.

\section{Recognition during the test phase}

Object-in-place performance in the CNQX+AP5 contra group was significantly impaired after a 5 min delay, compared with performance in the CNQX+AP5 ipsi group (mean discrimination ratio \pm SEM: $\mathrm{CNQX}+\mathrm{AP} 5$ contra, $0.056 \pm 0.078$; CNQX + AP5 ipsi, $0.53 \pm 0.08)$. ANOVA confirmed that there was a significant difference between the two groups $\left(F_{(1,8)}=\right.$ 23.77, $p<0.001)$. Further analyses revealed that the CNQX+AP5 ipsi group, but not the CNQX+AP5 contra, showed significant discrimination between the objects that had changed location compared with the objects in the same location $\left(\mathrm{CNQX}+\mathrm{AP5}\right.$ ipsi, $t_{(8)}=6.27, p<0.001 ; \mathrm{CNQX}+\mathrm{AP5}$ contra, $\left.t_{(8)}=0.68, p>0.05\right)$.

Exploration in sample and test phases

The CNQX+AP5 contra group did not differ from the CNQX+AP5 ipsi group in the amount of object exploration 
Table 2. Experiment 2: mean exploration times \pm SEM during the sample or test phase after bilateral infusions of vehicle or AP5 into the mPFC or PRH or unilateral infusions into both the PRH and $\mathrm{mPFC}$ in the object-in-place task ( 5 min delay)

\begin{tabular}{|c|c|c|c|c|c|c|c|}
\hline \multirow[b]{2}{*}{ Delay } & \multirow[b]{2}{*}{ Drug/side } & \multicolumn{2}{|l|}{$\mathrm{mPFC}$} & \multicolumn{2}{|l|}{ PRH } & \multicolumn{2}{|l|}{ Unilateral mPFC and PRH } \\
\hline & & Expl. in sample phase (s) & Expl. in test phase (s) & Expl. in sample phase (s) & Expl. in test phase (s) & Expl. in sample phase (s) & Expl. in test phase (s) \\
\hline \multirow[t]{4}{*}{$5 \mathrm{~min}$} & Veh & $53.7 \pm 4.4$ & $20.3 \pm 2.0$ & $61.3 \pm 3.6$ & $20.5 \pm 2.2$ & & \\
\hline & AP5 & $56.1 \pm 3.6$ & $17.5 \pm 2.3$ & $58.8 \pm 4.3$ & $20.9 \pm 2.4$ & & \\
\hline & Ipsi & & & & & $41.9 \pm 2.4$ & $12.6 \pm 1.8$ \\
\hline & Contra & & & & & $36.0 \pm 2.3$ & $12.2 \pm 1.4$ \\
\hline \multirow[t]{4}{*}{$1 \mathrm{~h}$} & Veh & $71.5 \pm 6.8$ & $25.5 \pm 2.6$ & $48.5 \pm 3.5$ & $16.2 \pm 1.9$ & & \\
\hline & AP5 & $68.3 \pm 3.4$ & $22.9 \pm 3.8$ & $50.0 \pm 2.65$ & $17.4 \pm 1.9$ & & \\
\hline & Ipsi & & & & & $54.9 \pm 4.4$ & $17.6 \pm 2.1$ \\
\hline & Contra & & & & & $49.9 \pm 2.5$ & $18.0 \pm 1.7$ \\
\hline
\end{tabular}

Expl., Exploration; Veh, vehicle.

completed in either the sample or test phase (sample phase, $F_{(1,8)}$ $=0.19, p>0.05$; test phase, $\left.F_{(1,8)}=0.23, p>0.05\right)$. The mean exploration values \pm SEM are as follows: sample phase: ipsi, $38.78 \pm 3.61$; contra, $40.11 \pm 2.27$; test phase: ipsi, $11.18 \pm 2.48$; contra, $12.79 \pm 1.96$.

\section{Experiment 4}

Experiment 3 confirmed that a non-NMDAR form of synaptic plasticity underlies short-term object-in-place information processing within the PRH. KARs in the PRH are known to be involved in short-term familiarity discrimination (Barker et al., 2006); thus, these receptors may also play an important role in short-term object-in-place associative memory. To test this hypothesis, the selective kainate $\left(\mathrm{GLU}_{\mathrm{K} 5}\right)$ receptor antagonist UBP302 was infused unilaterally into the PRH concomitantly with a unilateral infusion of AP5 into the ipsilateral (UBP + AP5 ipsi group) or contralateral (UBP+AP5 contra group) $\mathrm{mPFC}$.

\section{Recognition during the test phase}

Object-in-place performance in the UBP + AP5 contra group was significantly impaired compared with the UBP + AP5 ipsi group (mean discrimination ratio \pm SEM: UBP + AP5 contra, $-0.021 \pm 0.068$; UBP + AP5 ipsi, $0.37 \pm 0.057$ ), and ANOVA confirmed that there was a significant difference between the two groups $\left(F_{(1,7)}=13.61, p=0.01\right)$. Further analyses confirmed that the UBP + AP5 ipsi group showed significant discrimination $\left(t_{(7)}\right.$ $=6.05, p<0.01$ ), whereas the UBP $+\mathrm{AP} 5$ contra group did not $\left(t_{(7)}=0.27, p>0.05\right)$.

Exploration in sample and test phases

Analysis of the amount of exploration completed by the $\mathrm{UBP}+\mathrm{AP} 5$ contra and UBP+AP5 ipsi groups in either the sample or test phases revealed no significant differences (sample phase, $F_{(1,7)}=0.11, p>0.05$; test phase, $\left.F_{(1,7)}=0.22, p>0.05\right)$. The mean exploration values \pm SEM are as follows: sample phase: ipsi, $62.5 \pm 5.99$; contra, $59.63 \pm 2.69$; test phase: ipsi, $24.47 \pm 2.58$; contra, $27.89 \pm 3.18$.

\section{Experiment 5}

We have previously shown that disruption of NMDAR neurotransmission in the PRH impairs acquisition of familiarity discrimination after a $24 \mathrm{~h}$ delay but has no effect on acquisition or retrieval after a 20 min delay (Barker et al., 2006). In experiment 2 , a delay period of $1 \mathrm{~h}$ was used in the object-in-place task, so it was important to also investigate the effects of AP5 in the novel object preference task at the same delay. Therefore, the aim of experiment 5 was to investigate the effects of NMDAR blockade in the PRH on the acquisition and retrieval of familiarity discrimination after a 1 h delay.

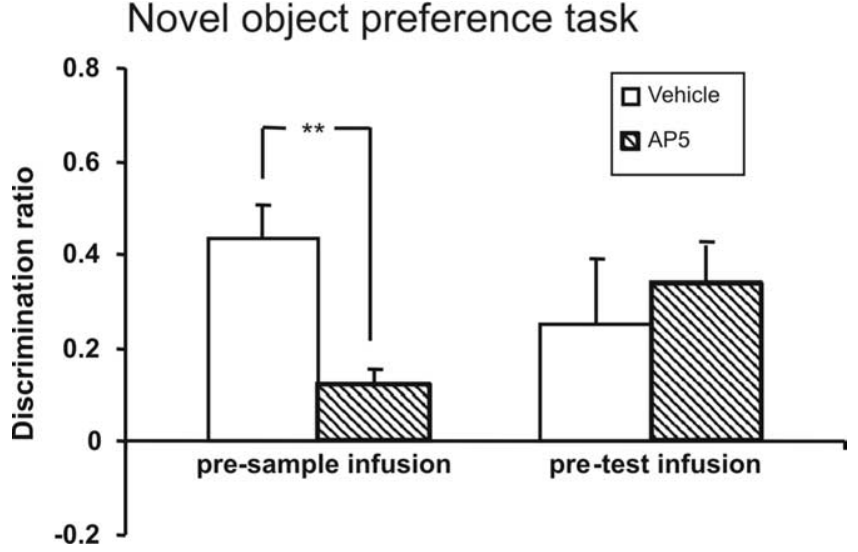

Figure 5. Novel object preference task. Perirhinal infusion of AP5 before the sample phase significantly impaired familiarity discrimination after a $1 \mathrm{~h}$ delay. ${ }^{* *} p<0.01$, difference between groups. In contrast, perirhinal infusion of AP5 before the test phase (right histogram) had no effect on familiarity discrimination after a $1 \mathrm{~h}$ delay.

Recognition during the test phase

Infusions of AP5 into the PRH before the sample phase significantly impaired familiarity discrimination after a $1 \mathrm{~h}$ delay compared with infusions of vehicle (Fig. 5) $\left(F_{(1,10)}=14.51, p<0.01\right)$. Further analyses revealed that rats infused with vehicle showed significantly greater mean relative exploration of a novel than a familiar object $\left(t_{(10)}=5.6, p<0.01\right)$. The mean discrimination ratio of the AP5-treated animals was significantly lower than the vehicle treated rats; however, the animals did show significant discrimination between the novel and familiar objects $\left(t_{(10)}=\right.$ $3.5, p<0.05)$. Infusion of AP5 before test had no effect on familiarity discrimination compared with vehicle $\left(F_{(1,7)}=0.2, p>\right.$ 0.05) (Fig. 5).

These results confirm that NMDAR neurotransmission is required for the acquisition of familiarity discrimination, but not for retrieval after a $1 \mathrm{~h}$ delay.

Exploration in sample and test phases

Analysis of the total amount of exploration completed in the sample or test phase revealed that infusion of AP5 into the PRH before the sample phase had no effect on the amount of exploration completed in this phase $\left(F_{(1,10)}=0.069, p>0.05\right)$ or in the amount of exploration in the test phase $\left(F_{(1,10)}=1.74, p>0.05\right)$. The mean exploration values \pm SEM are as follows: sample phase: vehicle, $23.0 \pm 5.6$; AP5, $23.3 \pm 5.0$; test phase, vehicle, $27.7 \pm 2.3 ; \mathrm{AP} 5,23.4 \pm 1.84$.

Infusions of AP5 or vehicle immediately before the test phase produced no significant differences in the amount of exploration completed $\left(F_{(1,7)}=0.048, p>0.05\right)$. The mean exploration val- 
ues \pm SEM are as follows: vehicle, $20.01 \pm 2.39 ;$ AP5, $19.28 \pm$ 2.60 .

\section{Discussion}

The present study sought to elucidate the neural basis of objectin-place associative recognition memory. The experiments confirmed that this memory process is dependent on a functional interaction between the PRH and MPFC, because performance was disrupted by simultaneous blockade of excitatory neurotransmission in both cortical regions by CNQX. In addition, it was demonstrated that acquisition of object-in-place memory, but not retrieval, is dependent on NMDAR-mediated neurotransmission in both the $\mathrm{MPFC}$ and PRH. Thus, acute intracerebral administration of AP5 into the MPFC before the sample phase of the object-in-place task impaired short and long-term memory performance, whereas intra-PRH AP5 impaired longterm memory only. Coadministration of AP5 into the PRH and mPFC in opposite hemispheres produced a significant impairment in long-term object-in-place memory, whereas short-term memory was unaffected. An infusion of CNQX into the PRH combined with NMDAR blockade in the MPFC impaired shortterm object-in-place memory, confirming that this memory process is dependent on information from the PRH over a short delay. Furthermore, a unilateral intra-PRH infusion of UBP302 combined with a unilateral infusion of AP5 into the contralateral $\mathrm{mPFC}$ significantly impaired object-in-place performance, highlighting, for the first time, the importance of KAR activation in the PRH for short-term object-in-place memory. The observed impairments produced by infusion of AP5 were in the acquisition of information, because memory was not impaired when infusions were made into either the PRH or $\mathrm{mPFC}$ immediately before the test phase.

CNQX infusions into the $\mathrm{MPFC}$ resulted in a greater amount of exploration, compared with vehicle infusions, in the test phase of experiment 1 . Such changes were not observed in the sample phase of this experiment. Thus, these increases in exploratory behavior may simply reflect the fact that the animals are treating all the object-in-place associations as novel in the test phase.

\section{The contribution of the perirhinal and prefrontal cortices to object-in-place associative memory}

In the object-in-place task, the animals learn which of four different objects occupies the four locations in the arena; thus, both object and place information must be acquired and then associated. Previous studies have demonstrated that such object-inplace associative memory may be dependent on several neural regions, including the PRH (Gaffan and Parker, 1996; Bussey et al., 2000, 2001; Barker et al., 2007) and the mPFC (Kesner and Ragozzino, 2003; Browning et al., 2005; Barker et al., 2007), and we have demonstrated previously (Barker et al., 2007) and confirmed in the present study that these two regions function as a neural circuit for object-in-place associative memory, with each region contributing to a distinct aspect of information processing. We have hypothesized, based on previous findings, that the $\mathrm{PRH}$ is responsible for the acquisition of the object information, whereas the $\mathrm{mPFC}$ is responsible for the integration of the object and location information (Rao et al., 1997; Barker et al., 2007).

Within the present experiment, we did not address the issue of which neural region might provide location information. One possibility is that such information is transmitted from the hippocampus to the $\mathrm{MPFC}$, and once the object and place information has been "tagged" as associated, this information is passed back to the hippocampus or to another region. Within the PRH-
mPFC circuit, we have therefore assumed that the primary direction of the flow of information, for the object-in-place task, is from temporal to frontal cortices. Thus, object information is initially processed by the PRH before being sent to the mPFC. This assumption is strongly supported by electrophysiological studies showing that the firing latencies of repetition sensitive neurons in the PRH are shorter than those in the mPFC (Xiang and Brown 2004). However, feedback from the mPFC to the PRH is also likely to be important within this neural system.

The present experiment demonstrated that infusion of AP5 into the $\mathrm{mPFC}$ impaired performance of the object-in-place task after both short and long retention delays, indicating that the integration of the object and place information, regardless of the length of delay period, must depend on mPFC NMDAR. This pattern of results contrasts with the effects observed after blockade of PRH NMDAR neurotransmission, where a behavioral impairment after long but not short delays was observed. However, the pattern of performance after intra-PRH infusions was strikingly similar to that observed previously using a novel object discrimination task (Barker et al., 2006). Thus, performance at short delays ( $5 \mathrm{~min}$ in the present object-in-place task and $20 \mathrm{~min}$ in the novel object preference task) was intact, whereas performance at longer delays ( $1 \mathrm{~h}$ in both tasks) was impaired.

We have argued previously that the AP5-induced delaydependent deficits in familiarity discrimination may reflect the operation of an NMDAR-dependent plastic process, which is slow to develop and long-lasting, hence not required at short delays, but crucial after long delays (Barker et al., 2006). Although it may be parsimonious to suggest a similar plastic process is involved in object-in-place memory, it must also be remembered that the two types of memory are dependent on different anatomical connections. Thus, object-in-place memory involves $\mathrm{mPFC}$ projections to the $\mathrm{PRH}$, whereas the $\mathrm{mPFC}$ is not necessary for familiarity discrimination.

\section{The role of NMDA receptor-mediated neurotransmission within the perirhinal-prefrontal circuit}

The present results clearly confirm the presence of an integrated neural circuit for object-in-place memory. Within the circuit, the PRH appears to be responsible for encoding the object information, whereas the $\mathrm{mPFC}$ is responsible for the integration of the object and the place information (the latter possibly provided by the hippocampus), so how may the delay-dependent effects of NMDAR blockade in the object-in-place task be accounted for?

Figure 6 provides an explanation of how infusions of AP5 into the $\mathrm{MPFC}$ and $\mathrm{PRH}$ produce different effects on object-in-place performance depending on the length of the retention delay used. When a short delay is imposed between sample and test, the unilateral intra-PRH AP5 infusion has little effect on the acquisition and processing of the "object" information, which is sent to the $\mathrm{mPFC}$ within the same hemisphere. At the same time, in the opposite hemisphere, the uninfused PRH will also send "object" information to the MPFC within the same hemisphere. Although here the blockade of mPFC-NMDAR will disrupt the integration of the object and location information unilaterally (AP5 infusions into the mPFC disrupted performance after a short and long delay), such unilateral disruption is not sufficient to disrupt behavior (Fig. 6a). Further support for this latter statement is provided by the finding that in all our experiments, ipsilateral infusions into the $\mathrm{PRH}$ and $\mathrm{mPFC}$ produced no behavioral impairments.

When a long delay is introduced between the sample and test phases, our results suggest that the neuronal processing required 


\section{a SHORT DELAY}

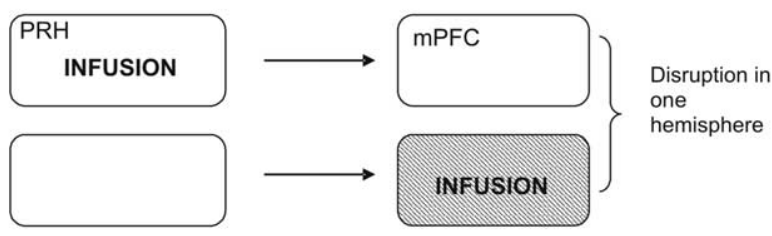

b LONG DELAY

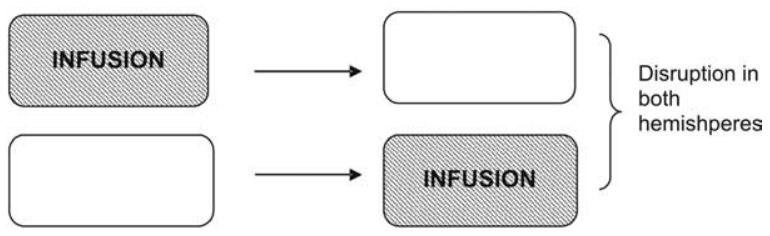

Figure 6. The effect of unilateral AP5 infusions into the PRH and $\mathrm{mPFC}$ on object-in-place performance. $\boldsymbol{a}$, AP5 infusion into the PRH has no effect on object processing after a short retention delay; thus, the disruption only occurs unilaterally within the neural network, and behavioral performance remains intact. $\boldsymbol{b}$, AP5 infusion into the PRH disrupts object processing after a long delay. As the network is disrupted bilaterally, performance in the object-in-place task is impaired.

to maintain object memory is disrupted by the infusion of AP5 into the PRH. Hence, either no object information is sent to the mPFC or a disrupted signal is sent; thus, the integration of the object and place information within the $\mathrm{MPFC}$ will not be normal. At the same time, in the opposite hemisphere, the PRH that has not been infused with AP5 may process the object information normally, yet the functioning of the MPFC in that hemisphere will have been disrupted by the unilateral AP5 infusion. In summary, the processing of object information and the integration of the object and place information is disrupted in both hemispheres, with the result that the formation of long-term object-in-place memory is impaired (Fig. 6b).

The present results show that the maintenance of long-term but not short-term object-in-place memory requires NMDA receptor activation in both the PRH and $\mathrm{mPFC}$, whereas KAR activation in the $\mathrm{PRH}$ is crucial for short-term object-in-place performance. This result, which parallels our previous findings into the neurochemical basis of familiarity discrimination (Barker et al., 2006), highlights the importance of KARs in a specific form of plasticity responsible for recognition memory over short delays, the precise nature of which requires further detailed study.

The putative role of other brain regions, such as the hippocampus, within the circuit still awaits examination. Future studies must also address the plasticity mechanisms underlying the integration of object and place information within the mPFC. Recent studies show that the MPFC contains neurons that significantly increase firing in response to familiar compared with novel stimuli, suggesting that increases in MPFC neuronal activation are critically associated with learning (Xiang and Brown, 2004). In light of these electrophysiological findings, a mechanism such as long-term potentiation may provide the best hypothesis for the change underlying neuronal response increments observed during object-in-place learning in the mPFC. Studies of this hypothesis will further help to elucidate the neural substrates of object-in-place associative learning within the PRH and mPFC. In conclusion, these data show for the first time that within a neural circuit for object-in-place associative memory, the PRH and mPFC cortices make distinct cognitive contributions, mediated by different underlying plastic processes.

\section{References}

Attwell PJ, Rahman S, Yeo CH (2001) Acquisition of eyeblink conditioning is critically dependent on normal function in cerebellar cortical lobule HVI. J Neurosci 21:5715-5722.

Barker GRI, Warburton EC, Koder T, Dolman NP, More JCA, Aggleton JP, Bashir ZI, Auberson YP, Jane DE, Brown MW (2006) The different effects on recognition memory of perirhinal kainate and NMDA glutamate receptor antagonism: implications for underlying plasticity mechanisms. J Neurosci 26:3561-3566.

Barker GRI, Bird F, Alexander V, Warburton EC, (2007) Recognition memory for objects, place and temporal order: a disconnection analysis of the role of the medial prefrontal cortex and perirhinal cortex. J Neurosci 27:2948-2957.

Browning P, Easton A, Buckley M, Gaffan D (2005) The role of prefrontal cortex in object-in-place learning in monkeys. Eur J Neurosci 22:3281-3291.

Bussey T, Duck J, Muir J, Aggleton J (2000) Distinct patterns of behavioural impairments resulting from fornix transection or neurotoxic lesions of the perirhinal and postrhinal cortices in the rat. Behav Brain Res 111:187-202.

Bussey TJ, Dias R, Amin E, Muir JL, Aggleton JP (2001) Perirhinal cortex and place-object conditional learning in the rat. Behav Neurosci 115:776-785.

Dix S, Aggleton J (1999) Extending the spontaneous preference test of recognition: evidence of object-location and object-context recognition. Behav Brain Res 99:191-200.

Ennaceur A, Delacour J (1988) A new one-trial test for neurobiological studies of memory in rats. 1: Behavioral data. Behav Brain Res 31:47-59.

Ennaceur A, Neave N, Aggleton J (1996) Neurotoxic lesions of the perirhinal cortex do not mimic the behavioural effects of fornix transection in the rat. Behav Brain Res 80:9-25.

Gaffan D, Murray E (1992) Monkeys (Macaca fascicularis) with rhinal cortex ablations succeed in object discrimination learning despite $24 \mathrm{~h}$ intertrial intervals and fail at matching to sample despite double sample presentations. Behav Neurosci 106:30-38.

Gaffan D, Parker A (1996) Interaction of perirhinal cortex with the fornix fimbria: memory for objects and "object-in-place" memory. J Neurosci 16:5864-5869.

Izquierdo L, Barros D, Ardenghi P, Pereira P, Rodrigues C, Choi H, Medina J, Izquierdo I (2000) Different hippocampal molecular requirements for short- and long-term retrieval of one-trial avoidance learning. Behav Brain Res 111:93-98.

Kesner R Ragozzino M (2003) The role of the prefrontal cortex in objectplace learning: a test of the attribute specificity model. Behav Brain Res 146:159-165.

Martin J (1991) Autoradiographic estimation of the extent of reversible inactivation produced by microinjection of lidocaine and muscimol in the rat. Neurosci Lett 127:160-164.

Mitchell J, Laiacona J (1998) The medial frontal cortex and temporal memory: tests using spontaneous exploratory behaviour in the rat. Behav Brain Res 97:107-113.

Murray E, Bussey T (1999) Perceptual-mnemonic functions of the perirhinal cortex. Trends Cogn Sci 3:142-151.

Rao SC, Rainer G, Miller EK (1997) Integration of what and where in the primate prefrontal cortex. Science 276:821-824.

Shi CJ, Cassell MD (1999) Perirhinal cortex projections to the amygdaloid complex and hippocampal formation in the rat. Comp Neurol 3406:229-328.

Watkins JC, Krogsgaard-Larsen P, Honoré T (1990) Structure-activity relationships in the development of excitatory amino acid receptor agonists and competitive antagonists. Trends Pharmacol Sci 11:25-33.

Winters BD, Bussey TJ (2005) Glutamate receptors in perirhinal cortex mediate encoding, retrieval, and consolidation of object recognition memory. J Neurosci 25:4243-4251.

Xiang J, Brown M (2004) Neuronal responses related to long-term recognition memory processes in prefrontal cortex. Neuron 42:817-829. 